factors within that particular home and dynamic. However, due to the small numbers of cases of SIDS in the time frame reviewed it was impossible to determine whether the inclusion of social care involvement to the SBS would improve the statistical model overall.

\section{PREVALENCE AND CLINICAL PRESENTATION OF NEONATAL THROMBOCYTOPENIA IN NEONATAL INTENSIVE CARE UNIT, SUEZ CANAL UNIVERSITY HOSPITAL}

Hazem Helmy Elemam. UK

\subsection{6/bmjpo-2021-RCPCH.14}

Background Thrombocytopenia is the commonest haematological abnormality encountered in the neonatal intensive care unit (NICU). The incidence in neonates varies greatly, depending on the population studied.

Objectives The aim of the present study was to study the prevalence of thrombocytopenia in the neonates admitted to the NICU.

Methods The study was carried out in 97 consecutive eligible neonates; we collected data from neonates admitted in Neonatal intensive care units (NICUs), Suez Canal university hospital in the period from April 2019 to October 2018

Platelets counts were done twice to avoid sample errors Results The prevalence of thrombocytopenia in the study group was 38.14\% (37/97). The prevalence of Mild- moderate thrombocytopenia was $21.64 \%(21 / 97)$ cases and the prevalence of severe thrombocytopenia group was $16.49 \%$ (16/97). Factors associated with thrombocytopenia were sepsis (75.67\%), pre-clampsia $(8.1 \%)$ in mothers. The most common bleeding manifestation was Interventricular haemorraghe $(16.2 \%)$. The overall mortality in babies with thrombocytopenia was $14.43 \%, 10$ neonates $(27.02 \%)$ with severe thrombocytopenia needed more than one time transfusion while 2 patients $(5.40 \%)$ only with mild thrombocytopenia needed platelet transfusion

Conclusions It is concluded that thrombocytopenia is very common in the NICU and should be actively looked for so that it can be managed appropriately.

\section{CONGENITAL SYPHILIS IN A 4-MONTH-OLD INFANT WITH LIMB WEAKNESS}

Elizabeth Morrisroe, Syeda Farah Farzana, James McKinnon. UK

10.1136/bmjpo-2021-RCPCH.15

Background Each year, syphilis complicates over 2 million pregnancies worldwide and continues to rise. Whilst still uncommon, rates of congenital syphilis have been rising in developed countries over recent years. In 2018 alone, 1,306 cases were reported in the United States; constituting a 185\% increase since 2014.

This disease can lead to peri-/neonatal death, prematurity and low birth weight. Untreated, spontaneous abortion can occur in $40 \%$ cases. Affected individuals often suffer longterm sequelae such as visual loss, sensorineural hearing loss, bone/joint deformity and neurodevelopmental delay.

Cases of congenital syphilis are uncommon in the UK; with an estimate of 10 cases annually in 2015, meaning most paediatricians will have little to no experience with the disease. Many affected individuals may be asymptomatic at birth and symptoms can often mimic other conditions. In this respect, and with rising incidence in adult disease over recent years, congenital syphilis poses a true diagnostic challenge for clinicians; as demonstrated in this case report.

Objectives In this report, we describe a rare case of a 4-monthold girl presenting with a several month history of reduced movement to the left arm accompanied by a maculopapular rash to the limbs. X-Ray findings included inflammatory periosteal changes to the radius and ulna. Blood test results included raised inflammatory markers (CRP and ESR) with a slight microcytic anaemia, raised ALP and suppressed TSH.

Methods

Results Treponema pallidum immunoglobin $\mathrm{M}$ (IgM) was detected in both baby and mother and a diagnosis of congenital syphilis was made.

Conclusions This case is an interesting clinical picture with a variety of important differential diagnoses including non-accidental injury, malignancy, autoimmune disease and other congenital infections. With an increasing rate of congenital syphilis infection in the developed world, it is vital that clinicians are able to recognise symptoms to ensure prompt diagnosis and treatment. In this respect, we can attempt to avoid the chronic and potentially life-threatening complications of untreated infection.

\section{PILOT STUDY DEMONSTRATES THAT PLACENTAL HISTOPATHOLOGY CAN BE A POTENTIAL ADDITIONAL TOOL FOR DIAGNOSING EARLY-ONSET NEONATAL SEPSIS}

ljas Hassan, Madhu George, ABDUL Thawab. India

\subsection{6/bmjpo-2021-RCPCH.16}

Background Early Onset Neonatal Sepsis (EONS) is one of the commonest and serious problem for newborn babies, incidence in India being 38 per 1000 intramural live births. Currently EONS diagnosis is mainly considered on maternal risk factors and non-specific neonatal symptoms. Also, initiating emperical antibiotics in timely fashion is critical in decreasing morbidity and mortality. But since a gold standard test is unavailable for EONS especially in the initial hours of life, number of neonates who require antibiotics is high. This inturn results in increase in disadvantages related to the antibiotic use and prolonged hospital stay.

We tried to explore whether placental histology can be used as an additional tool in diagnosing EONS thereby avoid the unnecessary antibiotic usage and hospital stay for neonates.

Objectives To determine whether placental histology has good correlation with probable or proven early-onset neonatal sepsis (EONS), thereby help to diagnose EONS and guide in clinical decision-making.

Methods This retrospective observational study comprised 101 newborn babies, both term and preterm, who were admitted to the neonatal intensive care unit of Rajagiri Hospital, Aluva, Kochi, Kerala, India between June 2017 and June 2020. EONS was defined as maternal risk factors with clinical symptoms (Suspected EONS) plus raised serial C-reactive protein (CRP) $>10 \mathrm{mg} / \mathrm{L}$ and a positive (Proven EONS) or a negative (Probable EONS) blood culture. Placentae were studied for a 